\title{
Archéopages
}

Archéopages

Archéologie et société

38 | 07/2013

Terres inhospitalières

\section{Un terroir réputé inhospitalier. Les occupations de la Sologne du Paléolithique au XIX ${ }^{\mathrm{e}}$ siècle}

\section{Philippe Salé}

\section{OpenEdition}

\section{Journals}

Édition électronique

URL : https://journals.openedition.org/archeopages/489

DOI : 10.4000/archeopages.489

ISSN : 2269-9872

\section{Éditeur}

INRAP - Institut national de recherches archéologiques préventives

\section{Édition imprimée}

Date de publication : 1 août 2014

Pagination : $12-19$

ISSN : $1622-8545$

\section{Référence électronique}

Philippe Salé, « Un terroir réputé inhospitalier. Les occupations de la Sologne du Paléolithique au xIx ${ }^{e}$ siècle », Archéopages [En ligne], 38 | 07/2013, mis en ligne le 01 juillet 2015, consulté le 22 janvier 2022. URL : http://journals.openedition.org/archeopages/489 ; DOI : https://doi.org/10.4000/ archeopages. 489 


\section{Un terroir réputé inhospitalier Les occupations de la Sologne du Paléolithique au $x x^{e}$ siècle}

Philippe Salé Inrap, UMR 7324 «Cites, Territoires, Environnement tet Sociétess"

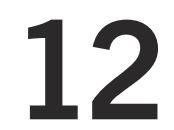

1. Loir-et-Cher : $68,7 \%$, Loiret : $18,8 \%$ et Cher : $12,5 \%$.

2. Selon la symbolique du Bureau de recherches géologiques et minières (BRGM)

\section{Le territoire}

La Sologne est une région naturelle de près de 500 ooo hectares, située dans le centre de la France et délimitée par les vallées de la Loire au nord et à l'ouest, du Cher au sud, et les marnes du Sancerrois vers l'est [ill. 1]. Ce territoire ne semble jamais avoir constitué une unité administrative (Poitou, 2009, p. 3). Dès la période antique, il est divisé entre les cités carnutes et bituriges, puis à partir de la première moitié du $\mathrm{IV}^{\mathrm{e}}$ siècle, entre le pagus Aurelianensis (chef-lieu : Aurelianum/ Orléans) et le pagus Bituricus (chef-lieu : Avaricum/Bourges). Aux périodes médiévales, cette répartition persiste puisque les diocèses reprennent au moins en partie le tracé des frontières antiques. Sur le plan féodal, la Sologne est répartie entre de multiples seigneuries, appartenant notamment au roi et au comte de Blois. Pour les périodes historiques, elle est donc toujours restée une zone frontalière, ce qui a pu impacter sur la nature des occupations. De nos jours, les départements du Loiret, du Loir-et-Cher et du Cher se partagent de manière inégale ce territoire'.

Géologiquement, la Sologne est une vaste cuvette formée dans la partie supérieure des calcaires de l'ère secondaire et comblée, à l'ère tertiaire, de sédiments d'origine granitique issus du Massif central (Fleury, 1992 [ill. 2]). Ils correspondent à des sables grossiers et des argiles qui se sont déposés sur une épaisseur pouvant atteindre plusieurs dizaines de mètres $\left(\mathrm{m}-\mathrm{p}^{2}\right.$, sables et argiles de Sologne). Ils sont complétés de sables grossiers déplacés par les cours d'eau au quaternaire et formant parfois des systèmes de terrasses (alluvions Fw, Fx, Fy et Fz). Au final, les couches rencontrées en surface peuvent être composées de sables grossiers purs ou d'argile compacte et présenter toutes les variétés entre ces deux formes. En l'absence de calcaire, les sols sont pauvres, acides et souvent peu épais. L'eau s'échappe des sédiments les plus sableux qui s'assèchent rapidement, alors qu'elle est retenue en surface par des sédiments argileux.

Le paysage de Sologne a beaucoup évolué depuis le début du XVIII ${ }^{\mathrm{e}}$ siècle (Heude, 2012). À cette date, il est largement dominé par les landes de bruyères et de genêts où paissent des moutons. Les terres cultivées sont rares, et il convient ensuite de les laisser reposer pendant 3, 6 ou 9 ans, voire plus, afin qu'elles se régénèrent. Ce n'est qu'à partir du Premier Empire, puis au Second, que les plantations d'arbres se développent et donnent le paysage que l'on connaît actuellement. Il se caractérise par des espaces boisés associés à de grands domaines, par la présence de nombreux étangs et par un habitat dispersé : essentiellement des villages et de rares habitats isolés. C'est un territoire qui contraste énormément avec les régions alentour : la Loire (la « vallée des rois »), la Beauce (le « grenier de la France »), la Touraine («le jardin de la France »), mais également le Berry.

\section{La Sologne, une terre de désolation selon les érudits des $\mathrm{XVIII}^{\mathrm{e}}$ et $\mathrm{XIX}^{\mathrm{e}}$ siècles}

Les caractéristiques des sols de Sologne semblent contraindre la population à des conditions de vie particulièrement difficile. Aux XVIII ${ }^{\mathrm{e}}$ et XIX ${ }^{\mathrm{e}}$ siècles, plusieurs auteurs donnent une piètre image de ce pays et de ses habitants. Ainsi, en 1811, PierreMarie-Sébastien Bigot de Morogues témoigne du pauvre état de la population (Bigot de Morogues, 1811). Les Solognots sont peu nombreux, chétifs et malingres. « Leurs membres sont grêles, et leur corps voûté de fort bonne heure : souvent leur teint est pâle et quelquefois jaunâtre. Les enfants surtout s'y font remarquer par la grosseur de leur ventre, parfois accompagnée de la bouffissure de la 


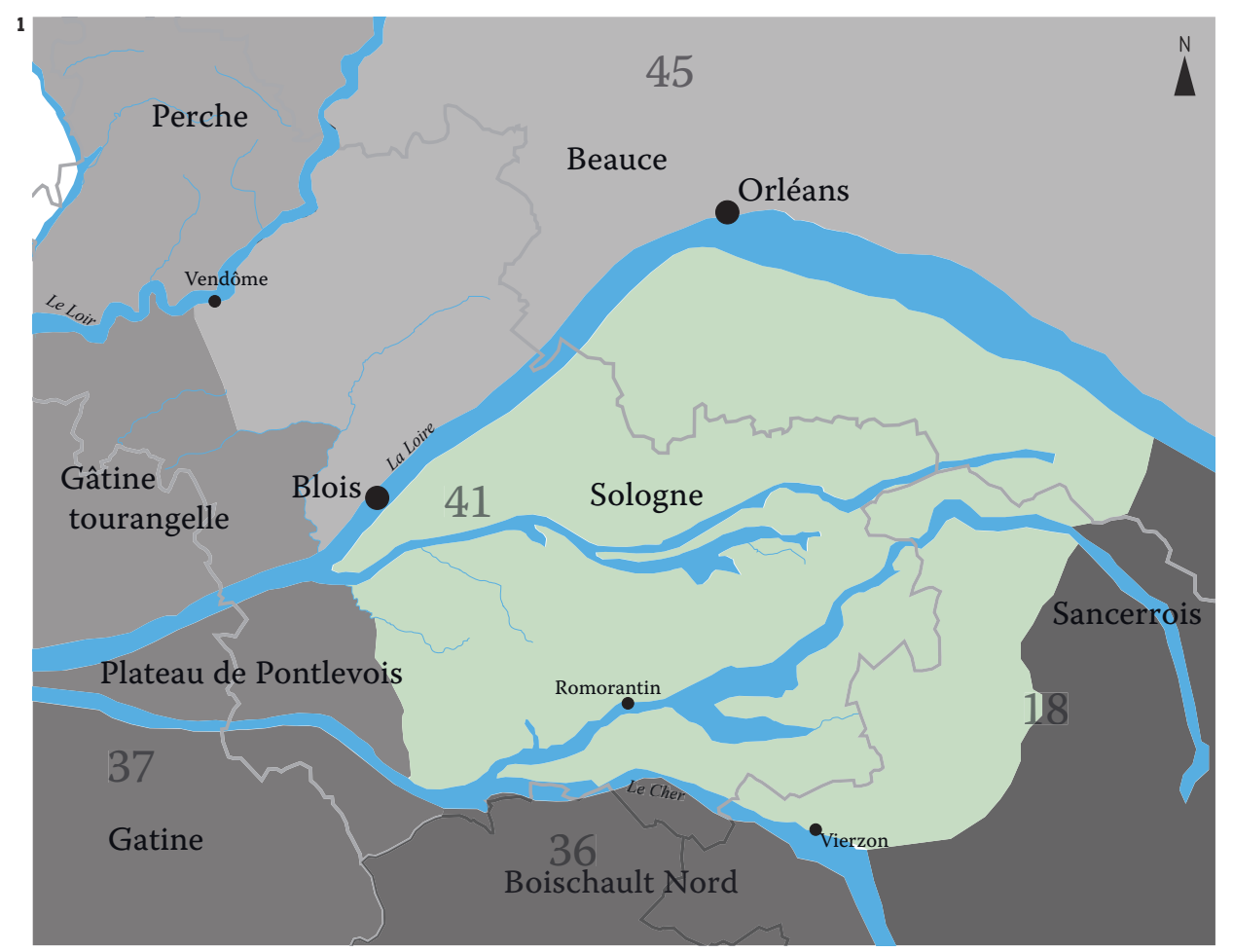

1. La Sologne entre Loire et Cher.

2. La géologie de la

Sologne d'après l'atlas

des paysages du Loir-et-

Cher (carte géologique

simplifiée, conseils

d'architecture,

d'urbanisme et de

l'environnement du

Loir-et-Cher: http://www.

atlasdespaysages.caue41

fr/images/hd/cartel0bis.

jpg/, consulté le

30/09/2013).
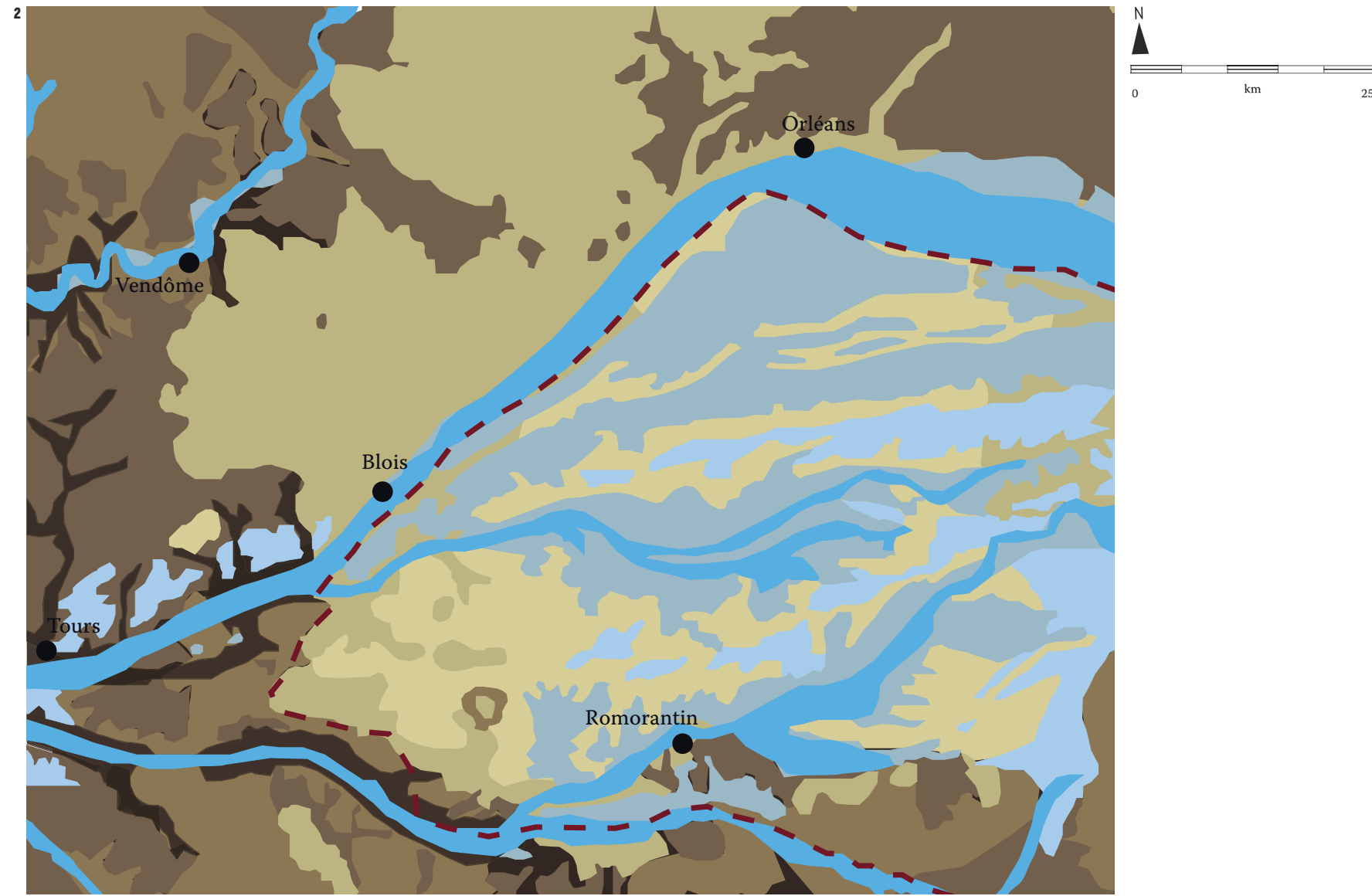

- Villes principales

- - - Contours Sologne

Alluvions récentes

Alluvions anciennes

Sables et argiles de Sologne (Miocène)

Calcaires lacustres de Beauce et de Touraine

(Oligocène/Miocène)

Calcaires lacustres (Eocène)

Argiles à silex (Crétacé)

Craie à silex et tuffeau (Crétacé) 


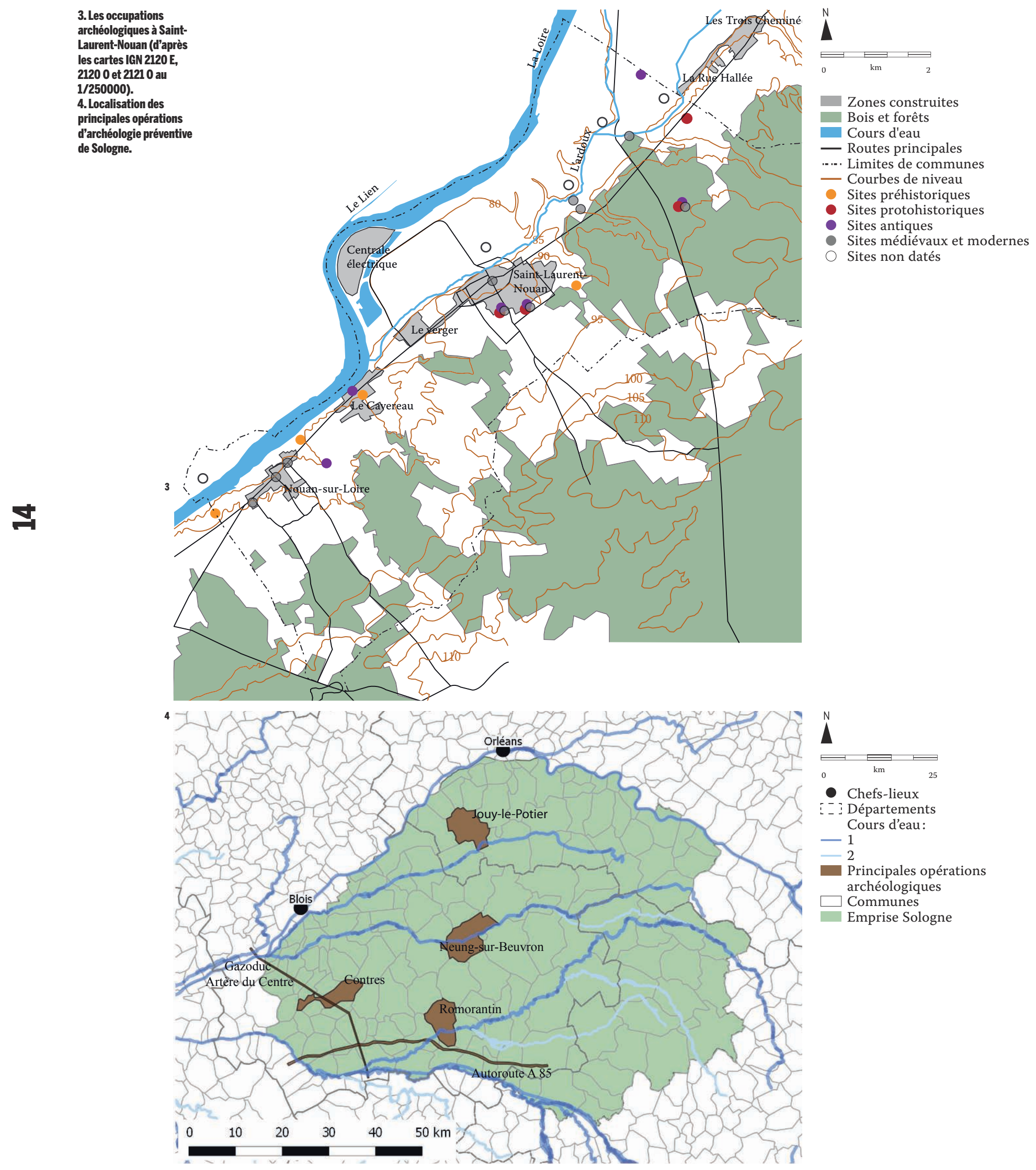


face $[. .$.$] ; la dentition ne se fait qu'avec peine et$ détermine de terribles convulsions [...] ; la croissance très lente ne se termine qu'avec peine [...]. » En 1892, Louis Gallouédec dépeint également un tableau bien sombre : « Les hydropisies, le rachitisme et les scrofules décimaient du reste les habitants de la Sologne : ceux qui n'en mouraient point restaient estropiés. La misère était générale. Point de fermes pour habitations, mais des huttes chétives, des locatures, quelques misérables morceaux de bois soutenant un mur d'argile d'un double centimètre d'épaisseur... » (Gallouédec, 1892, p. 385).

Les difficultés semblent plus importantes en Sologne qu'en bien d'autres lieux de France aux $\mathrm{XVIII}^{\mathrm{e}}$ et XIX ${ }^{\mathrm{e}}$ siècles. Ainsi, le paludisme, transmis par les moustiques dont les larves se développent dans les eaux stagnantes des étangs, paraît particulièrement virulent (Heude, 1982). De même, la dysenterie semble conduire une partie de la population de la vie au trépas (Viaud, 1986). Même les animaux sont touchés : Pierre-Marie-Sébastien Bigot de Morogues mentionne des épizooties régulières, comme « la maladie rouge » (Bigot de Morogues, 1811). Les landes sont également favorables au développement des loups : jusqu'à la fin du XIX ${ }^{\mathrm{e}}$ siècle, des battues sont organisées pour tuer ces animaux (Barré, Delétang, 1984). Souvent, les érudits relatent une histoire où les populations ne font que survivre. Louis Gallouédec écrit : « tout le passé de cette région ne comprend qu'une suite de vicissitudes pendant lesquelles elle passe d'une prospérité toujours assez médiocre... à la plus profonde misère » (Gallouédec, 1892, p. 384).

\section{Des sources incertaines?}

L'examen des données archéologiques permet de vérifier si la Sologne a toujours été une terre inhospitalière. Mais il n'est pas envisageable de détailler ici chaque période. Nous nous contenterons donc d'effectuer des éclairages ponctuels, en fonction des travaux déjà réalisés. Par ailleurs, les sites placés en bord de Loire et du Cher n'ont pas été retenus, en raison de la spécificité de ces axes majeurs de communication. La répartition des sites sur les communes qui se développent sur les deux terroirs (vallées et Sologne), à l'image de SaintLaurent-Nouan [ill. 3], montre en effet un très net déséquilibre en faveur des occupations à proximité des cours d'eau.

Les données archéologiques collectées dans la base Patriarche ${ }^{3}$ sont principalement issues des prospections anciennes ou plus récentes et des travaux de l'archéologie préventive. Or la couverture forestière du sol de Sologne peut entraver la réalisation de prospections pédestres et aériennes. De plus, en l'absence de ville importante, les aménagements nécessitant la réalisation de diagnostics sont moins nombreux. Ces deux facteurs pourraient donc expliquer la faible densité des sites. Mais la Sologne est un territoire qui a fait l'objet de prospections dès la première moitié $d u$ XIX $^{\mathrm{e}}$ siècle alors que le pays était encore couvert de landes (M. de La Saussaye), puis de nombreux chercheurs se sont succédé : MM. Martinet Florance, Delétang, Amelin, Magiorani et d'autres ont activement œuvré pour la connaissance du patrimoine archéologique solognot. Par ailleurs, si les opérations d'archéologie préventive sont moins nombreuses que le long de la Loire par exemple, on peut cependant constater une activité régulière. Plusieurs grands tracés linaires ont été réalisés dans la région, mais seuls l'A $85^{4}$ et le gazoduc Artère du Centre ${ }^{\mathbf{5}}$ ont donné lieu à des diagnostics et des fouilles. Par ailleurs, les projets d'aménagement ont été particulièrement suivis par les conservateurs du SRA Centre sur les communes de Contres, Neung-sur-Beuvron et Romorantin (Loir-et-Cher) et Jouy-le-Potier (Loiret) [ill. 4]. Enfin, l'image que donnent à voir ces opérations semble corréler l'impression générale que laisse la carte archéologique de ce territoire. Mais elle est nettement plus contrastée que la présentation des auteurs du XIX ${ }^{\mathrm{e}}$ siècle.

\section{Des données archéologiques variables selon les époques}

Pour les périodes les plus anciennes, les données sont disparates et peu abondantes, et concernent essentiellement des objets isolés. Ainsi, sur l'A85 (Krausz, 2003) et le gazoduc Artère du Centre (Salé, 1999 et 2001), seuls quelques outils isolés attribuables au Paléolithique ont été enregistrés. Récemment, un lot de silex daté du Paléolithique moyen a été découvert à Contres (Gransar, à paraître), mais il semble déplacé. Pour le Paléolithique supérieur, il semble que « le grand paléo-lac miocène qui a conduit à la mise en place du plateau de Beauce et de la Sologne au cœur de la région n'est resté qu'un espace de passage » (Agogué, 2005, p. 519). En effet, les principaux sites recensés pour cette période se situent en périphérie de ces deux territoires. Aucune synthèse n'est encore publiée pour le Mésolithique en région Centre et les occupations concernées sont peu nombreuses. Pour la Sologne, un seul site a été fouillé : il est situé sur le tracé de l'A85, à SaintRomain-sur-Cher, et concerne plusieurs campements successifs attribués au Mésolithique ancien et moyen (Kildea, 2008). Les autres sites de cette période sont localisés le long de la Loire ou du Cher.

Les données régionales concernant le Néolithique sont présentées dans le catalogue de l'exposition de 1999 portant sur les premiers paysans en région Centre (Agogué, Leroy, Verjux, 1999 [ill. 5]). Pour la Sologne, les vestiges sont concentrés à l'ouest. Aucune construction monumentale (fossés ou chapelets de fossés) n'a été découverte. À Thenay, en revanche, on a retrouvé la seule tombe du Néolithique ancien de la région. Deux sites d'habitat sont également mentionnés, mais le premier, à Châtres-sur-Cher, serait plus vraisemblablement daté de l'âge du Bronze (Krausz, 2003, p. 40). Le second est attribué à la culture Chambon et est situé à Contres,
3. Base de données
archéologiques natio

Valaire) n'a livré que

des indices ténus : quat

Paléolitribués au

du mobilier erratique

à Billy et une fosse à Feings pour la Protohistoire :

un ensemble de structures

avec scories et céramiques médiévales à Contres.

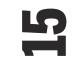


5. Les occupations néolithiques en région Centre (d'après Agogué, Leroy, Verjux, 1999, p. 14 42, 92).

6. Les tertres funéraires en Sologne (d'après Milcent, 1995, p. 66).

$\mathscr{9}$
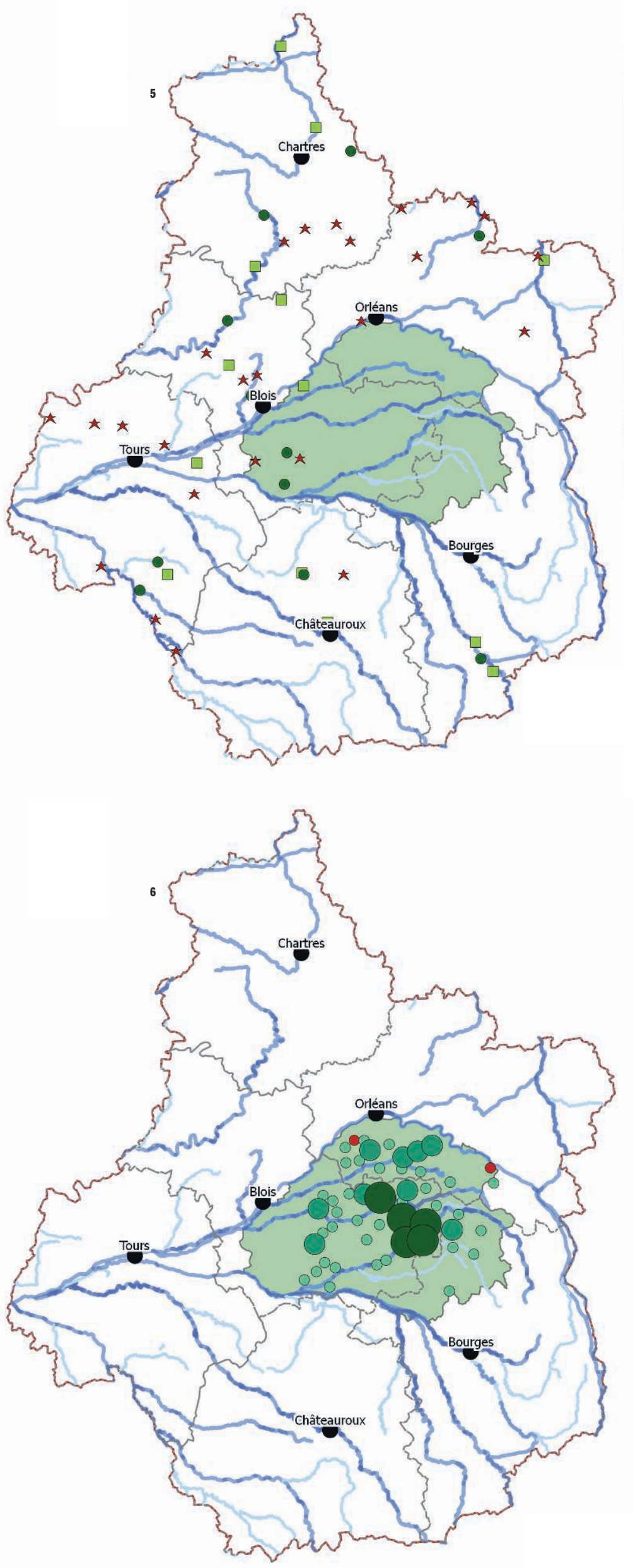

I

0

Sites néolitiques

Constructions monumentales

- Habitat

$\star$ Funéraire

Chefs-lieux

1
-

Cours d'eau:

Départements

$\square$ Région

$\square$ Emprise de la Sologne

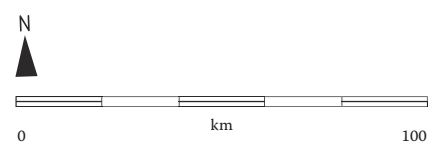

Tumuli:

- Unique et imposant

1 à 10

11 à 20

21 et plus

Chefs-lieux

Cours d'eau :

$-1$

Départements

$\square$ Région

$\square$ Emprise de la Sologne 
au Château-Gabillon (Loir-et-Cher). À Méhers, sur l'A85 (ibid., p. 33), une grande quantité d'outils taillés et de céramiques est datée du Néolithique récent ou final, mais aucune structure n'est associée. On notera également qu'à Soings-en-Sologne quatre fosses, découvertes lors d'un diagnostic et datées du Néolithique récent au Bronze ancien, pourraient correspondre à des incinérations (De Filippo, 2013).

Pour la Protohistoire, les mentions sont plus nombreuses, et concernent en majorité des ensembles funéraires. On en recense dans presque toutes les communes de Sologne : il s'agit souvent de quelques tertres, mais ils forment parfois de véritables nécropoles qui atteignent plusieurs dizaines d'individus (Milcent, 1995) [ill. 6]. Plus de 500 monuments sont signalés, mais très peu d'entre eux ont été fouillés et ils sont donc rarement datés. La découverte de mobilier isolé peut évoquer une occupation de la Sologne dès le début de l'âge du Bronze (ibid., p. 45). Dans la deuxième partie $\mathrm{du}$ Bronze moyen, les mentions sont plus nombreuses, mais elles sont concentrées vers le nord et le nord-est et à l'extrémité occidentale du pays. La documentation concernant le Bronze final est plus riche. Elle est constituée essentiellement de sites à vocation funéraire ou de dépôts localisés principalement sur les marges de la Sologne, plus rarement au centre. On signalera en particulier la fouille des tumulus à Mennetou-sur-Cher sur l'A85 (Salanova (dir.), 1999). Les sites d'habitat recensés dans le cadre de « l'enquête Bronze » sont plus rares, mais couvrent toute la période (Frénée et al., à paraître) : l'un, situé à Méhers, est daté du Bronze ancien ; un grand bâtiment du Bronze moyen est localisé à Châtres-sur-Cher ${ }^{\mathbf{6}}$; un troisième, du Bronze final, a été mis au jour à Contres (Froquet, 2012). D'autres sites, plus nombreux, sont situés dans les vallées du Cher ou de la Loire.

Pour l'âge du Fer, la majorité des données est également issue de sites funéraires ou de dépôts. Pour le premier âge du Fer, Pierre-Yves Milcent a étudié ces données dans le cadre d'une thèse universitaire qui a fait l'objet d'une publication (Milcent, 2004). Ce travail montre que, dans le centre de la France, cette période connaît un processus d'évolution en trois grandes étapes. Les sites de Sologne, relativement bien documentés, participent pleinement à cette évolution. Les habitats sont moins bien connus et aucun site du premier âge du Fer n'est cité dans les derniers travaux synthétiques (Buchsenschutz, Fichtl, 2008). Cependant, quelques fouilles récentes commencent à combler cette lacune. Ainsi, deux sites ont été découverts à Méhers sur le tracé de l'A85 (Krausz, 2003, p. 46-48) et deux autres sont également connus à Romorantin sur la ZAC des Grandes Bruyères (Froquet, 2002). Ils correspondent à des petites unités domestiques aux datations différentes, mais qui représentent toutes les phases de la période. Au second âge du Fer, quelques sites funéraires ont été découverts au nord de la Sologne
(Marion et al., 2011), mais les mentions d'habitats sont plus nombreuses. À Neung-sur-Beuvron, les nombreux diagnostics réalisés sont liés à la présence d'un oppidum et d'une agglomération secondaire antique. Des sites d'habitat ruraux sont également connus en plusieurs endroits : par exemple à Méhers, sur le tracé de l'A85 (Krausz, 2003, p. 46-48), à Contres (Froquet, 2012), ou à SaintLaurent-Nouan (Salé, Gransar, Gay, à paraître)...

Pour l'Antiquité, Neung-sur-Beuvron et Neuvysur-Barangeon sont les seules agglomérations secondaires de Sologne qui soient certifiées. Les sites de Vouzon, Soings-en-Sologne et Souesmes sont plus incertains ou mal documentés (Cribellier, 2012). Les autres agglomérations (Gièvres, Thésée, Saint-Romain-sur-Cher, Vienne-en-Val...) sont en bordure de la Loire ou du Cher. Dans le cadre de l'atlas 2000 sur le Berry antique, la moitié est de la Sologne du Loir-et-Cher a été intégrée à l'enquête qui regroupe toutes les mentions connues de la carte archéologique (Batardy, Buchsenschutz, Dumasy, 2001). Ce document montre que la densité des occupations rurales est nettement plus faible en Sologne que dans les autres pays environnants. De plus, aucun site n'est enregistré comme villa. Les différents travaux d'archéologie préventive semblent confirmer cet aperçu : les fouilles de la ZAC des Grandes Bruyères à Romorantin (Joly, 2007) ou celles de l'A85 (Krausz, 2003) montrent des implantations modestes : des éléments parcellaires (Pruniers-enSologne) ; des constructions isolées (Saint-Romainsur-Cher, Gy-en-Sologne, deux à Romorantin) ; ou peu nombreuses (deux bâtiments à Méhers) ; et des n64écropoles qui paraissent faiblement développées (un petit ensemble à Gy-en-Sologne dont les limites restent inconnues et de rares fosses à incinération à Billy). Plus récemment, à Soings-enSologne, quelques sépultures, qui pourraient être rattachées à un établissement périphérique à l'agglomération, ont été découvertes (De Filippo, 2013).

Pour le début du haut Moyen Âge, Grégoire de Tours ne cite aucun vici, castra ou castellum pour la Sologne (Hervé, 1999), ni aucun monument religieux (Vieillard-Troiekouroff, 1976). En revanche, Henri Delétang fait état de centaines de fortifications en terre, enceintes, maisons fortes et mottes pour des périodes plus récentes (Delétang, 1984, vol 2, p. 15 ; Delétang, 2008). Mais de manière générale, les travaux de synthèse font défaut pour cette période. Les fouilles d'archéologie préventive restent également peu nombreuses : à Contres, une nécropole des $\mathrm{VI}^{\mathrm{e}}$ et $\mathrm{VII}^{\mathrm{e}}$ siècles a été découverte récemment (Dalayeun, 2013), deux sites d'habitats ruraux ont été fouillés sur l'A 85 (Krausz, 2003), l'un à Pruniers-en-Sologne, daté du $\mathrm{x}^{\mathrm{e}}$ ou XI ${ }^{\mathrm{e}}$ siècle, et l'autre à Mennetou-sur-Cher (à proximité d'un chemin), attribué au XIII ${ }^{\mathrm{e}}$ siècle ; à Contres de nouveau, un ensemble de structures, sur le gazoduc Artère du Centre (Salé, 2001) ; et à Lassaysur-Croisne, un site fossoyé (Munos, à paraître).

Le réseau des agglomérations est peu dense au
6. Bien qu'en Sologne ce site soit placé dans une zon restreinte où affleurent de marnes lacustres tertiaires. 
bas Moyen Âge (Galinié, Audinet, 1993) : Romorantin reste une petite ville, malgré les projets grandioses de François $\mathrm{I}^{\mathrm{er}}$ et Léonard de Vinci, qui resteront sans suite ; Contres apparaît comme une agglomération de troisième rang. À Jouy-le-Potier, plusieurs diagnostics archéologiques ont été menés ces dernières années en raison notamment de la présence d'ateliers de potiers datés du XIII ${ }^{\mathrm{e}}$ au XVI ${ }^{\mathrm{e}}$ siècle (Méténier, Jesset, 2006). À noter également qu'aucun couvent mendiant n'est construit à la fin du Moyen Âge en Sologne (Galinié, 1993). On peut supposer que le paysage rural devait probablement présenter des caractéristiques très différentes de celles d'aujourd'hui, mais plus proches des descriptions du XVIII ${ }^{\mathrm{e}}$ siècle : de vastes domaines fonciers couverts en grande partie de landes, peu propices aux activités agricoles hormis l'élevage ovin ; des étangs, dont la création pourrait remonter principalement aux $\mathrm{XV}^{\mathrm{e}}$ et $\mathrm{XVI}^{\mathrm{e}}$ siècles (Delétang, 1984, vol. 1, p. 66); des villages relativement distants les uns des autres et de rares habitats isolés.

\section{Des occupations inégalement développées}

Les auteurs qui ont étudié la Sologne évoquent fréquemment les disparités de la documentation, liées à l'absence de ville importante et à la couverture végétale forestière et aux biais qu'elles impliquent. Les recherches qui se succèdent depuis le début du XIX ${ }^{\mathrm{e}}$ siècle donnent effectivement une image particulière des occupations de la Sologne. L'étude de ce territoire souffre probablement de la rareté des fouilles, en particulier au centre et à l'est, mais les diverses opérations d'archéologie préventives réalisées depuis ces quinze dernières années semblent confirmer le bilan des prospections.

Le peuplement de la Sologne paraît débuter tardivement (au Néolithique et peut-être au Mésolithique), mais pour les périodes anciennes les données restent rares. À partir de lâge du Bronze, les occupations sont en revanche nettement plus nombreuses. Il est encore difficile de préciser à partir de quelle date ce territoire connaît un dynamisme. Il est également peu évident de connaître son origine : est-il lié par exemple à un déplacement de population ou à une forte croissance naturelle? Quoi qu'il en soit, au Bronze final le réseau des occupations semble montrer une forte densité, à l'image de celles de la commune de Contres. Ce dynamisme semble se poursuivre pendant tout le premier âge du Fer. À la période suivante, des occupations sont toujours attestées, mais celles des régions environnantes, comme la Beauce par exemple, semblent plus nombreuses et plus ostentatoires. C'est donc peut-être dès La Tène que le déclin s'amorce : le développement des agglomérations en Sologne ou sur ces marges (vallées de la Loire et du Cher), et la recherche de terres plus riches en sont peut-être les raisons. À la période antique, les occupations existent bien sûr (des exploitations rurales, et des agglomérations secondaires), mais elles forment un réseau peu dense et les occupations rurales correspondent majoritairement à de petites unités. Vers la fin du haut Moyen Âge et jusque vers le XIII ${ }^{\mathrm{e}}$ siècle, la région semble connaître un sursaut d'activité, avec la construction de nombreuses fortifications en terre (liée à son rôle de zone frontière?). Mais il est probable que depuis la fin du Moyen Âge, la situation soit proche de celle décrite par les auteurs $\mathrm{du} \mathrm{XIX}^{\mathrm{e}}$ siècle : un territoire peu productif et des populations pauvres. 

continuités et ruptures de l'occupation territoriale au Paléolithique supérieur en région Centre », Bulletin de la Société préhistorique française, t. 102, $\mathrm{n}^{\circ} 3$, p. 509-526.

Agogué O., Leroy D., VerjuX C., 1999 : Les premiers paysans en région Centre (50oo-200o av. J.-C.), Catalogue d'exposition, Musée des beaux-arts d'Orléans, 1999-200o, Orléans, Arep, 119 p.

Barré D., Delétang H., 1984 : « Les loups à Souesme (Loir-et-Cher) au XIX ${ }^{\mathrm{e}}$ siècle », Bulletin du Groupe de recherches archéologiques et historiques de Sologne, 4, 1, p. 93-122.

Batardy C., Buchsenschutz O., Dumasy F., 2001 : «Le Berry antique. Milieu, hommes, espaces, atlas 2000 ", Revue archéologique du centre de la France, 21, $190 \mathrm{p}$.

Bigot DE Morogues P.-M.-S., 1811 : « Essai sur la topographie de la Sologne et sur les principaux moyens d'amélioration qu'elle présente ", Société des sciences physiques, médicales et d'agriculture d'Orléans, 3, p. 181-211.

Buchsenschutz O., Fichtl S., 2008 : « Les Celtes dans la boucle de la Loire ", Les dossiers d'archéologie, 326, p. 2-5.

CRIBEllier C., 2012 : « Trois siècles de découvertes et trois décennies de recherches sur les agglomérations antiques de la région Centre », in Cribellier C., Ferdière A. (dir.), Agglomérations secondaires antiques en région Centre, Actes de la table ronde d'Orléans, 18-19 novembre 2004, Tours, FERACF (coll. Suppl. à la RACF, 22), p. 9-26.

DaLAYEUn M., 2013 : Diagnostic archéologique sur l'extension de la zone d'activités Le Grille Midi, Rapport de diagnostic, SRA Centre/Inrap, Tours, $70 \mathrm{p}$.

De FILIPpo R., 2013 : Soings-en-Sologne, Loir-et-Cher, Le Sauveur, Rapport de diagnostic, SRA Centre/Inrap, Tours, $93 \mathrm{p}$.

DELÉTANG H., 1984: Voirie antique et occupation du sol en Sologne, le chemin gallo-romain d'Orléans à Bourges, Thèse de doctorat, Université de Tours, 3 vol.

DELÉTANG H., 2008: "Mottes médiévales de la vallée de la Sauldre (Cher et Loir-et-Cher) », Bulletin du Groupe de recherches archéologiques et historiques de Sologne, 3o, 4, p. 63-76.

FleUry R., 1992 : «Salbris », in Carte géologique de la France au 1/50ooo, Orléans, Bureau de recherches géologiques et minières.

Frénée E., Froquet-Uzel H., Hamon T., Mercey F, NoËL J.-Y. (à paraître) : « L'habitat et l'occupation du sol à lâge du Bronze et au premier âge du Fer », in Carozza L., Marcigny C., Talon M., L'habitat et l'occupation du sol à l'âge du Bronze et au premier âge du Fer : restitution de l'enquête nationale, Actes de la table ronde de Bayeux, 29-30 novembre 2011.

FROQUET H., 2002 : Romorantin-Lantenay (Loir-etCher), ZAC Les Grandes Bruyères, Document final de synthèse d'évaluation préventive, SRA Centre/ Inrap, Tours, $22 \mathrm{p}$.

Froquet H., 2012: Contres, Loir-et-Cher. Un habitat protohistorique à vocation agro-pastorale, Rapport final d'opération, SRA Centre/Inrap, Tours, 148 p.

GALINIÉ H., 1993 : « Les villes et les agglomérations secondaires au bas Moyen Âge : le Loir-et-Cher $\mathrm{XIV}^{\mathrm{e}}-\mathrm{XV}^{\mathrm{e}}$ siècle », in GALINIÉ H. et Royo M. (dir.), Atlas des villes et des réseaux de villes en région Centre, Tours, Association en région Centre pour l'histoire et l'archéologie.

GALinié H., Audinet I., 1993 : « Les villes, les agglomérations secondaires et les couvents mendiants, $\mathrm{XIII}^{\mathrm{e}}$ - $\mathrm{XV}^{\mathrm{e}}$ siècle », in GALINIÉ H. et Royo M. (dir.), Atlas des villes et des réseaux de villes en région Centre, Tours, Association en région Centre pour l'histoire et l'archéologie.

GAllouéDeC L., 1892 : « La Sologne », Annales de géographie, t. $1, \mathrm{n}^{\circ}$ 4, p. 379-389.

Gransar M. (à paraître) : La Fosse des Roches à Contres, Loir-et-Cher, Rapport d'opération de diagnostic SRA Centre/Inrap, Tours.

HERVÉ C., 1999 : «Liste des vici, castra, castella cités par Grégoire de Tours en région Centre», in BELLET M.-E., Cribellier C., Ferdière A. et Krausz S. (dir.), Agglomérations secondaires antiques en région Centre, Tours, FERACF (coll. Suppl. à la RACF, 17), p. 219-220.

Heude B., 1982 : « À propos du paludisme en Sologne... et ailleurs ", Bulletin du Groupe de recherches archéologiques et historiques de Sologne, 4, 1, p.12-20.

HEUDE B., 2012 : La Sologne, des moutons, des landes, des hommes. XVIII siècle-Second Empire, Rennes, Presses universitaires de Rennes (coll. Histoire), $360 \mathrm{p}$.

Joly S., 2007 : Romorantin-Lanthenay et Villefranchesur-Cher. Saint-Martin et Les-Terres-Fortes,

Rapport final d'opération d'évaluation archéologique, SRA Centre/Inrap, Tours, 21 p.

KildeA F., 2008: « Les occupations du Mésolithique ancien et moyen de Saint-Romain-sur-Cher (Loiret-Cher) », in Fagnart J.-P., ThÉvenin A., Ducroce T., Souffi B., Coudret P. (dir.), Le début du Mésolithique en Europe du Nord-Ouest, Actes de la table ronde d'Amiens, 9-10 octobre 2004. Paris, éd. Société préhistorique française (coll. Mémoire, 15), p. 156-167.

KRAUSZ (dir.), 2003 : Les fouilles sur l'autoroute A85, entre Theillay et Saint-Romain-sur-Cher, Catalogue d'exposition, Musée de Sologne, Les Amis du Musée de Sologne, 80 p.

Marion S., Gaultier M., Villenave C., Chimier J.-P., 2011: « Sépultures et ensembles funéraires du second âge du Fer en Île-de-France et en région Centre ", in Barral P., Dedet B., Delrieu F., Giraud P., Le Goff I., Marion S., Villard-LeTIEC A. (dir.), Gestes funéraires en Gaule au second âge du Fer, Actes du XXXIII colloque de l'Association française pour létude de l'âge du Fer, Caen, 20-22 mai 2009, Besançon, Presses universitaires de Franche-Comté (coll. Environnement, sociétés et archéologie, 14).

MÉTÉNIER F., JeSSET S., 2006 : « Bilan des connaissances sur l'activité potière médiévale à Jouy-le-Potier (Loiret) », Revue archéologique du Loiret, 29 p. 55-74.

MilCEnT P.-Y., 1995: « La Sologne entre Bronze et Fer $\left(\mathrm{xIII}^{\mathrm{e}}-\mathrm{V}^{\mathrm{e}} \mathrm{s}\right.$. av. J.-C.) ", in DelétANG H., Bulletin $d u$ Groupe de recherches archéologiques et historiques de Sologne, 17, 3, p. 45-73.

Munos M. (dir.), à paraître : Lassay-sur-Croisne, Le Bourg, un site fossoyé médiéval en Sologne, Rapport de fouille archéologique préventive, SRA Centre/ Inrap, Tours.

Poitou C., 2009: « Les limites de la Sologne du XVI ${ }^{\mathrm{e}}$ au $\mathrm{xx}^{\mathrm{e}}$ siècle », Bulletin du Groupe de recherches archéologiques et historiques de Sologne, 31, 3 , p. 1-12.

Salanova L. (dir.), 1999 : Autoroute A85 Tours-Vierzon Le monument $n^{\circ} 1$ de la nécropole tumulaire des Barres à Mennetou-sur-Cher, Document final de synthèse de fouille préventive, SRA Centre/Afan, Romorantin, $127 \mathrm{p}$.

SALÉ P. (dir.), 1999 : Canalisation de transport de gaz Artère du Centre, section Chémery (Loir-et-Cher) Roussines (Indre), Rapport d'une opération préventive de fouille d'évaluation, SRA Centre/ Afan, Orléans, 2 vol.

SALÉ P. (dir), 2001: Canalisation de transport de gaz Artère du Centre, section Chaumont-sur-LoireChémery (Loir-et-Cher), Rapport d'une opération préventive de fouille d'évaluation, SRA Centre/ Afan, Orléans, 2 vol.

SALÉ P., Gransar M., GAY J.-P. (à paraître) : « Un établissement rural à enclos fossoyé de La Tène $\mathrm{C}_{2} /$ D1 en Val de Loire : le site du Vivier à Saint-LaurentNouan (Loir-et-Cher) », Revue archéologique du centre de la France.

VIEILlARD-TROIEKOUROFF M., 1976 : Les monuments religieux de la Gaule d'après les œuvres de Grégoire de Tours, Paris, Champion, $491 \mathrm{p}$

VIAUD D., 1986 : « Sur la mort en Sologne dans la deuxième moitié du XVII ${ }^{\mathrm{e}}$ siècle. L'exemple de Tremblevif (Saint-Viâtre, Loir-et-Cher) », $L a$ Sologne et son passé, 8, 4, p. 115-130. 